\title{
Assessments of mental capacity in psychiatric inpatients: a retrospective cohort study
}

\author{
Penelope F Brown ${ }^{1 *}$, Alex D Tulloch², Charlotte Mackenzie³ ${ }^{3}$ Gareth S Owen ${ }^{1}$, George Szmukler ${ }^{2}$ \\ and Matthew Hotopf ${ }^{1}$
}

\begin{abstract}
Background: The Mental Capacity Act 2005 (MCA) was introduced in 2007 to protect vulnerable individuals who lack capacity to make decisions for themselves and to provide a legal framework for professionals to assess incapacity. The impact of the MCA on clinical practice is not known. This study aims to evaluate how frequently mental capacity is assessed in psychiatric inpatients, whether the criteria for determining capacity set out in the MCA are used in practice, and whether this has increased with the introduction of the MCA.

Method: A retrospective cohort study was carried out using a case register of South East London mental health service users. The Case Register Interactive Search (CRIS) system enabled searching and retrieval of anonymised information on patients admitted to the South London and Maudsley NHS Foundation Trust since 2006. The presence and outcomes of documented mental capacity assessments in psychiatric admissions between May 2006 and February 2010 were identified and demographic information on all admissions was retrieved.
\end{abstract}

Results: Capacity assessments were documented in 1,732/17,744 admissions (9.8\%). There was a significant increase in the frequency of capacity assessments carried out over the study period of 0.3 percentage points per month ( $95 \% \mathrm{Cl} 0.26-0.36, \mathrm{p}<0.00001)$. In only $14.7 \%$ of capacity assessments were the MCA criteria for assessing capacity explicitly used.

Conclusions: Over the period of the introduction of the MCA there has been a significant increase in the number of mental capacity assessments carried out on psychiatric inpatients. Although mental health services are considering the issue of capacity more frequently, mental capacity assessments are inconsistently applied and do not make adequate use of MCA criteria.

\section{Background}

The Mental Capacity Act 2005 (MCA) came into force in October 2007 and codifies in statute the common law principles of assessing and managing individuals who lack certain decision-making capacities in England and Wales. It is expected that all doctors and other health professionals are able to assess capacity according to the guidance set out in the MCA, or the Adults with Incapacity (Scotland) Act 2000, and accompanying Codes of Practice. The test for incapacity is laid out in section 2 MCA and involves two stages. Firstly, to lack capacity the individual must be found to suffer from "an impairment of or disturbance in the functioning of the brain or

\footnotetext{
* Correspondence: penelope.brown@kcl.ac.uk

'Department of Psychological Medicine, Institute of Psychiatry, Weston Education Centre, Cutcombe Road, London SE5 9RJ, UK

Full list of author information is available at the end of the article
}

mind". Secondly, the person must be unable to make the relevant decision at the relevant time because of that impairment, due to lacking one or more of the following abilities: (1) the ability to understand and (2) retain information relevant to the decision; (3) the ability to use or weigh that information as part of the process of making the decision; and (4) the ability to communicate their decision [1].

The introduction of the MCA reflects a general shift in social policy changes and attitudes, especially within healthcare settings, regarding the importance of autonomous decision making and has led to increased interest in how mental capacity is conceptualized and assessed [2-5]. However, there is very little research on how and when capacity assessments are performed in practice, and studies in general medical inpatients suggest capacity assessments are often carried out when the patient 
is refusing treatment or is difficult to manage [6,7]. Whether there has been a change since the introduction of the MCA is not known. One question that remains unclear despite recent legal reform is how frequently capacity assessments should be performed. A key principle of the MCA is that capacity should be presumed, but when should this presumption be called into question? Incapacity is common in medical inpatients, with over $30 \%$ estimated to lack capacity to make treatment decisions, and is often not recognised by clinicians [8]. In psychiatric inpatients the prevalence of incapacity is higher $(40-60 \%[9,10])$ and it has been suggested that assessment of capacity should form a core part of inpatient psychiatric assessment [10]. Whether this is being done in practice is not known.

When capacity should be assessed in psychiatric settings is particularly unclear due to the existence of the Mental Health Act 1983 (MHA). While this provides a legal framework for detaining and treating patients for their psychiatric needs without their consent, and without consideration of whether they have capacity or not, the MHA still requires healthcare professionals to determine whether a person has the capacity to consent to or refuse treatment. The MHA Code of Practice (para 23.27-29) states that all assessments of capacity must be recorded in the patients' notes, and that the capacity of patients with mental disorders can fluctuate and capacity assessments should be made at the time the decision in question needs to be made [11]. Even where treatment under the MHA does not require the patient's consent, for example medical treatment given to a patient within 3 months of detention under section $3 \mathrm{MHA}$, it is recommended that the patient's consent should nevertheless be sought, and their capacity to consent recorded [12] (para 23.37). In addition, for patients detained under the provisions of the MHA, the assessment of capacity is explicitly required in certain specified circumstances, most notably when electro-convulsive therapy (ECT) is offered, or when medication is prescribed after three months. In such instances the medical practitioner must certify in writing that the patient is not capable of understanding the "nature, purpose and likely effects of that treatment" ([13] section 58) and a second opinion must be sought before treatment can be given. In informal psychiatric inpatients (i.e. those not detained under the MHA) an assessment of capacity is fundamental to determining whether the safeguards within the MCA including the rules on restraint (as laid out in sections 5 and $6 \mathrm{MCA}$ ) and the 'Deprivation of Liberty Safeguards' (introduced by the Mental Health Act 2007) - apply.

Amid the current complexity in mental health law in England and Wales, the Healthcare Commission (now the Care Quality Commission, or CQC) have recommended routine assessment and recording of mental capacity to consent to treatments and interventions from the start of every informal psychiatric inpatient admission [14]. More recently the CQC has raised concerns that questionable assumptions about patients' capacity to consent to medication could lead to unlawful treatment in patients detained under the MHA [15]. They recommend good recording of capacity assessments regarding consent to treatment, including a record of the discussions that lie behind the outcome of the assessment [12]. This guidance suggests that, despite the principle of assumed capacity, capacity assessments should be carried out as a matter of routine on all psychiatric inpatients, whether informal or detained under the MHA, but this leads to significant questions about the resources available for such assessments and the subsequent quality of capacity assessments.

There have been no large-scale studies examining the impact of the MCA on clinical practice and the quantity and quality of capacity assessments being carried out. This study aims to evaluate how frequently mental capacity is assessed in psychiatric inpatients, whether the criteria for determining capacity set out in the MCA are used, and whether there has been a change in practice with the introduction of the MCA. We use data from the South London and Maudsley NHS Foundation Trust (SLaM) using its Biomedical Research Centre Case Record Interactive Search, a novel informatics system which allows free text in electronic records to be searched for research purposes [16]. We report the frequency of capacity assessments carried out on psychiatric inpatients and how this has changed over recent years, testing the hypothesis that the implementation of the MCA has led to an increase in the number of capacity assessments being reported. We also examine whether clinicians are using the criteria specified in the MCA for determining incapacity, and the prevalence of incapacity in different patient groups.

\section{Methods}

\section{Setting and study population}

The SLaM BRC Clinical Record Interactive Search (CRIS) provides anonymised, in-depth information derived from electronic medical records relating to secondary mental health care, which includes all specialist care for hospitalization. The protocol for this system has been described elsewhere [16-18]. SLaM provides comprehensive secondary mental health care to a population of approximately 1.3 million residents of four London boroughs (Lambeth, Southwark, Lewisham and Croydon) as well as tertiary care national referral units. Electronic clinical records have been used comprehensively across all SLaM services since 2006 and the CRIS system was developed in 2008 to allow searching and retrieval of anonymised information with over 180,000 cases currently represented on 
the system. CRIS was approved as a dataset for secondary analysis by Oxfordshire Research Ethics Committee C, reference 08/H0606/71.

\section{Inclusion criteria}

We studied all admissions to SLaM psychiatric wards culminating in a discharge between $1^{\text {st }}$ May 2006 and $31^{\text {st }}$ January 2010, a forty-five month period spanning the introduction of the MCA. These comprised admissions to any inpatient service including older adults, child and adolescent mental health, forensic psychiatry, rehabilitation services and mental health in learning disability in any of the four London boroughs served by SLaM, as well as national specialist referral units (including eating disorders, psychosis and affective disorders units) during the study period were included. Individuals who were admitted on more than one occasion during the study period were counted for each admission, and information was gathered on each admission for the period of the inpatient stay only. Those less than sixteen years of age at the time of their admission were excluded as the MCA is only concerned with individuals aged sixteen years or over.

\section{Exposure variables}

For each admission, we extracted age at admission, gender, ethnicity, diagnosis (based on the 10th edition of the World Health Organization International Classification of Diseases (ICD-10)) and Mental Health Act status of each individual, as well as the type of service or ward they were managed under. When more than one diagnosis was recorded for an individual, the one given closest to date of discharge was used. When an admission had been under a number of sections of the Mental Health Act, the most restrictive section was used (e.g. if a patient had been detained under section 2 and under 3 in the same admission they were recorded as being detained under section 3).

\section{Capacity assessments}

The outcome of interest in this study was the documentation in the notes of a capacity assessment carried out at any time during the admission. The search terms "capacity" and "competence" were initially used to identify documented capacity, however, after preliminary analysis of 100 inpatient records, the term "competence" did not yield any additional assessments to the term "capacity", and this search term was subsequently dropped. All items of correspondence and progress notes containing the term "capacity" were manually reviewed by PB \& CM, and any uses of the term "capacity" which were not related to mental capacity assessments were discarded. For all records of mental capacity assessments, the following information was collected: the decision for which the capacity assessment was carried out ("decision type" e.g. the capacity to consent to medication or the capacity to make a will); whether the capacity assessment was carried out or just suggested by a member of the clinical team and not subsequently done; whether the MCA criteria for assessing capacity were documented; whether a specific document for recording the capacity assessment was used; and whether the patient had or lacked capacity in relation to the decision in question. For patients who had multiple capacity assessments during a single admission, only the first recorded assessment was used in the analysis.

Descriptive analysis of data investigating trends in capacity assessments was carried out using Stata 10. A time trend in the proportion of documented capacity assessments was analysed using linear regression after checking for auto-correlation with the Durbin-Watson test.

\section{Results}

\section{Main outcome measures}

17,744 psychiatric admissions of individuals aged 16 years or over (mean age 41.7, range 16-96 years, s.d 15.5) were identified during the study period (May 2006February 2010). Table 1 shows the clinical and legal characteristics of the study cohort. The outcome of interest (a documented capacity assessment) was present in $1,732(9.8 \%, 95 \%$ CI $9.3-10.2 \%)$ of admissions. In a further 423 (2.4\%), a mental capacity assessment was suggested by a member of the clinical team, but no record of the assessment taking place was documented. The majority of capacity assessments were carried out by doctors $(1,227 / 1,732,70.7 \%)$, with the remaining by approved social workers or approved mental health practitioners $(308 / 1,732,17.8 \%)$, nurses $(103 / 1,732,6.0 \%)$, and other members of the multidisciplinary team $(34 / 1,732$, $2.0 \%)$. In 60 assessments (3.5\%) the assessor's profession was unknown.

Capacity assessments were carried out for many different reasons, including capacity to consent to psychiatric admission and treatment, capacity to consent to marriage and divorce, capacity to make a will, capacity to refuse life-saving treatment and capacity to consent to sharing medical records. The numbers of capacity assessments per decision type are summarized in Table 2.

The criteria for determining mental capacity according to the MCA were reported in 254/1,732 capacity assessments (14.7\%). This increased from $11.5 \%$ before the implementation of the MCA to $15.5 \%$ after. This increase did not reach statistical significance (Chi-squared 3.718, $\mathrm{p}=0.052$ ); a similar borderline significant increase was observed using time series analysis ( 0.13 percentage points per month, $95 \% \mathrm{CI}$ : -0.007 to $0.268, \mathrm{p}=0.06$ ). In the majority of assessments no criteria, or arbitrary criteria, were reported. In only eight cases $(0.5 \%)$ was a 
Table 1 Personal, clinical and legal characteristics of psychiatric admissions during the study period

\begin{tabular}{llll}
\hline Category & $\begin{array}{l}\text { Number } \\
(\mathrm{n}=17,744)\end{array}$ & (\%) & $\begin{array}{l}\text { Number (\%) with } \\
\text { documented mental } \\
\text { capacity assessment }\end{array}$ \\
\hline
\end{tabular}

\begin{tabular}{llll}
\hline Gender & & & \\
Female & 8,147 & 45.9 & $824(10.1)$ \\
Male & 9,597 & 54.1 & $908(9.5)$ \\
Age Group & & & \\
$16-25$ & 2,629 & 14.8 & $295(11.3)$ \\
$26-35$ & 4,368 & 24.6 & $335(7.7)$ \\
$36-45$ & 5,184 & 29.2 & $376(7.2)$ \\
$46-55$ & 2,800 & 15.7 & $228(8.1)$ \\
$56-65$ & 1,188 & 6.7 & $150(12.7)$ \\
$66-75$ & 776 & 4.4 & $151(19.3)$ \\
$76+$ & 799 & 4.5 & $197(24.7)$
\end{tabular}

Ethnicity

White European

Black African

Black Caribbean

Black other

East Asian

South Asian

Mixed, other

Marital Status

Single

Married/civil partnership

Divorced/separated

Widowed

Not-known

\section{Diagnosis}

Organic and Developmental Disorders

Schizophrenia

10,511

$59.2865(8.2)$

1,913

$10.8269(14.1)$

1,567

$8.8 \quad 211(13.5)$

1,946

410

337

1,060

$11.0 \quad 212(10.9)$

$2.3 \quad 35(8.5)$

$1.9 \quad 29(8.6)$

$6.0111(10.5)$

Schizoaffective and other

Psychotic Disorders

Bipolar Disorder

$\begin{array}{lll}11,164 & 64.6 & 1,077 \\ (9.4)\end{array}$

$2,283 \quad 12.9 \quad 244(10.7)$

$2,182 \quad 12.3 \quad 188(8.6)$

$\begin{array}{llll}658 & 3.7 & 141(21.4)\end{array}$

$1,157 \quad 6.5 \quad 82(7.1)$

Depression and Neurotic Disorders

Personality Disorders $\quad 909$

Substance Misuse Disorders

Eating Disorders and Other

Behavioural Disorders

Unknown

Service

General Adult Services

PICU

1,957

$67.4 \quad 1,074(9.0)$

MHOA

$564 \quad 3.2 \quad 108(19.0)$

$1,390 \quad 7.8 \quad 328(23.7)$
Table 1 Personal, clinical and legal characteristics of psychiatric admissions during the study period (Continued)

\begin{tabular}{llll}
\hline CAMHS & 255 & 1.4 & $28(11.0)$ \\
Specialist & 746 & 4.2 & $81(10.9)$ \\
Learning Disabilities & 60 & 0.3 & $24(40.0)$ \\
Addictions & 2,527 & 14.2 & $16(0.6)$ \\
Forensic & 177 & 1.0 & $62(35.0)$ \\
Rehab & 68 & 0.4 & $11(16.2)$ \\
Mental Health Act Status & & & \\
Informal & 10,608 & 59.8 & $433(4.1)$ \\
Section 4/5/136 & 703 & 34.0 & $68(9.8)$ \\
Section 2 & 2,326 & 13.1 & $332(14.3)$ \\
Section 3* & 3,740 & 21.1 & $507(13.6)$ \\
Forensic & 367 & 2.1 & $92(25.1)$ \\
Total & $\mathbf{1 7 , 7 4 4}$ & $\mathbf{1 0 0}$ & $\mathbf{1 , 7 3 2}(\mathbf{9 . 8})$ \\
*Section 3 detained & $\mathbf{2 , 2 0 1}$ & 12.4 & $353(22.9)$ \\
for $>3$ months & & & \\
\hline
\end{tabular}

specific form for documenting mental capacity assessments used.

\section{Time trend analysis}

The frequency of capacity assessments was analysed by month from May 2006 to January 2010. In the first month, 5\% of admissions had an assessment; in the final month (January 2010) it was over 17\%. Time-series regression indicated a significant increase in the proportion of assessments carried out over the study period (see Figure 1) with no evidence of autocorrelation (Durbin-Watson statistic $=2.22$ ); the regression coefficient was 0.294 (95\% CI: 0.258 to $0.328, \mathrm{p}<0.0001$ ), showing a gradual increase in the proportion of approximately 0.3 percentage points per month. There was no step-wise increase in the proportion of inpatients assessed for capacity immediately after the introduction of the MCA in November 2007 (regression coefficient

Table 2 Frequency of capacity assessments per decision type

\begin{tabular}{lll}
\hline Decision type & $\mathbf{n}$ & \% \\
\hline Psychiatric admission & 752 & 43.4 \\
Psychiatric treatment including ECT & 435 & 25.1 \\
Aftercare and accommodation & 111 & 6.4 \\
Physical health interventions & 174 & 10.1 \\
Legal issues & 59 & 3.4 \\
Finances, contracts, LPA, AD & 75 & 4.4 \\
Other & 126 & 7.3 \\
Total & $\mathbf{1 , 7 3 2}$ & \\
\hline
\end{tabular}

(LPA = lasting power of attorney $A D=$ advanced directive). 


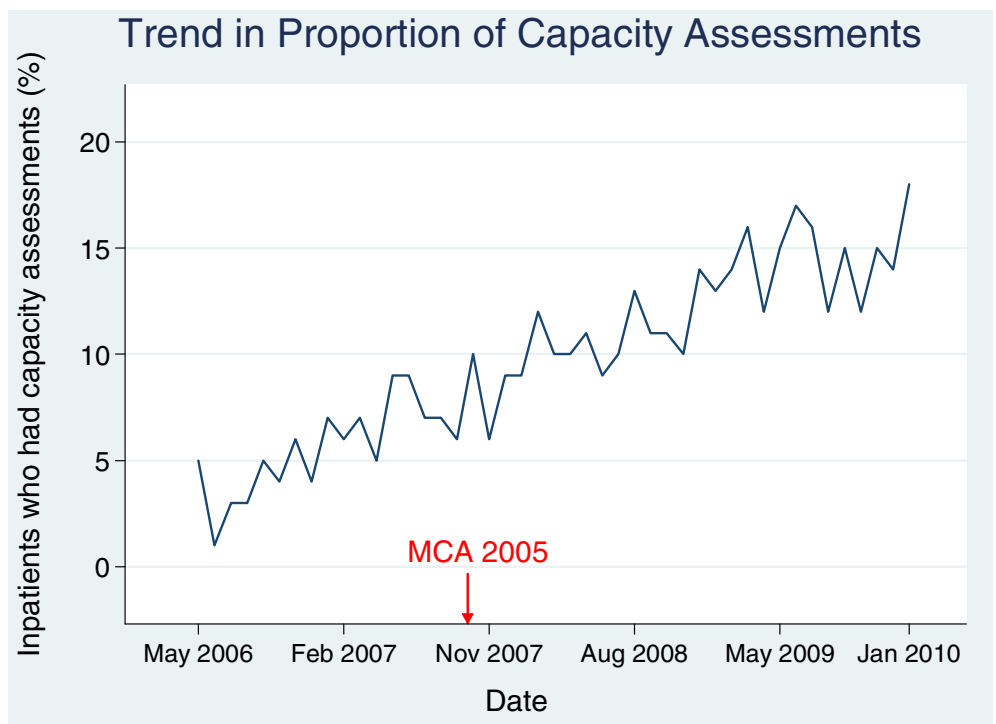

Figure 1 Time trend (per month) of the percentage of inpatients in whom capacity was assessed during their admission.

$0.59,95 \% \mathrm{CI}:-1.21$ to $2.39, \mathrm{p}=0.5)$, perhaps suggesting an anticipatory effect of the change in legislation.

\section{Outcomes}

Overall, $1,101(63.6 \%)$ of the admissions assessed for mental capacity were found to lack capacity. 612 (35.1\%) were reported to have capacity, and in $19(1.1 \%)$ of the reported capacity assessments no outcome or an ambiguous outcome (e.g. "fluctuating capacity") was given. The proportion of admissions lacking capacity varied by diagnosis (see Table 3) with organic and developmental disorders showing the highest prevalence $(82.2 \%$ and $67.1 \%$ respectively). Table 4 shows the proportion of psychiatric admissions found to lack capacity according to MHA status. Patients detained under civil sections of the MHA were more likely to lack capacity than those admitted to hospital voluntarily, however, over $50 \%$ of informal patients were found to lack capacity when assessed.

When the proportion of capacity assessments was broken down by month there was a significant decrease in the frequency of incapacity reported over the study period (regression coefficient $-0.427,95 \%$ CI -0.623 to $-0.230, \mathrm{p}=0.0001$ ) showing a reduction of 0.4 percentage points per month.

\section{Discussion}

For an individual to give their consent to treatment or a procedure they must have the mental capacity to do so, and it is crucial that healthcare professionals are able to assess capacity according to the MCA. Mental capacity should be assessed in relation to specific decisions taken

Table 3 Proportion of assessments found to lack capacity by diagnosis

\begin{tabular}{|c|c|c|c|}
\hline Diagnosis & $\begin{array}{l}\text { Number (\%) of admissions } \\
\text { with documented capacity } \\
\text { assessment }\end{array}$ & $\begin{array}{l}\text { Number assessed to } \\
\text { lack capacity }\end{array}$ & $\begin{array}{l}\%(95 \% \mathrm{Cl}) \text { assessed } \\
\text { to lack capacity }\end{array}$ \\
\hline Organic and Developmental & $236(26.0)$ & 194 & $82.2(77.3-87.1)$ \\
\hline Schizophrenia & $547(14.0)$ & 367 & $67.1(63.1-71.0)$ \\
\hline Schizoaffective/Other Psychotic & $268(12.7)$ & 163 & $60.8(54.9-66.7)$ \\
\hline Bipolar Disorder & $232(11.8)$ & 160 & $69.0(63.0-75.0)$ \\
\hline Depression and Neurotic Disorders & $211(6.5)$ & 111 & $52.6(45.8-53.4)$ \\
\hline Personality Disorders & $55(6.1)$ & 16 & $29.1(16.7-41.5)$ \\
\hline Substance Misuse Disorders & $109(3.0)$ & 47 & $43.1(33.7-52.6)$ \\
\hline Eating and other Behavioural Disorders & $20(8.1)$ & 6 & $30.0(8.0-52.0)$ \\
\hline Unknown & $54(6.1)$ & 37 & $68.5(55.7-81.3)$ \\
\hline Total & 1,732 & 1,101 & $63.6(61.3-65.8)$ \\
\hline
\end{tabular}




\begin{tabular}{|c|c|c|c|}
\hline MHA status & $\begin{array}{l}\text { Number (\%) } \\
\text { of admissions } \\
\text { with documented } \\
\text { capacity } \\
\text { assessment }\end{array}$ & $\begin{array}{l}\text { Number (\%) } \\
\text { assessed to } \\
\text { lack capacity }\end{array}$ & $\begin{array}{l}\%(95 \% \mathrm{Cl}) \\
\text { assessed to } \\
\text { lack capacity }\end{array}$ \\
\hline Informal & 637 & 320 & $50.3(46.3-54.1)$ \\
\hline S 4/5/136 & 186 & 149 & $80.1(74.3-85.9)$ \\
\hline S2 & 324 & 274 & $84.6(80.6-88.5)$ \\
\hline S3 & 507 & 342 & $67.5(63.4-71.5)$ \\
\hline Criminal section & 78 & 16 & $20.5(11.3-29.7)$ \\
\hline Total & 1,732 & 1,101 & $63.6(61.3-65.8)$ \\
\hline
\end{tabular}

at specific times. This study shows that, in psychiatric clinical practice, capacity is assessed in relation to a variety of decisions, ranging from whether an individual is able to consent to a blood test, to having ECT or to being placed in long-term care homes. We found a significant increase in the proportion of inpatients with documented assessments of capacity in relation to a number of treatment decisions over the period of the implementation of the MCA. This increase was observed both before and after the MCA came into force, suggesting that the legislation itself did not have decisive and immediate effects on practice but provoked anticipatory changes. It is also perhaps associated with a more general shift in culture and increased awareness of capacity issues for psychiatric inpatients. Despite this increase, capacity assessments are infrequently carried out with fewer than $10 \%$ of psychiatric inpatients having documented assessments for capacity during their admission.

It is not clear from the legal guidance whether mental capacity should be routinely assessed in all psychiatric inpatients, especially since a key principle of the MCA is that capacity should be presumed. Mental capacity is only of legal relevance in the MHA when obtaining consent to treatment in specific circumstances (ECT and medical treatment after 3 months when the patient is detained under section 3), although it should also be considered when dealing with issues not related to psychiatric treatment such as physical healthcare and welfare issues. Its importance in informal inpatients is less clear and it could thus be argued that assessments of capacity in other circumstances, including informal inpatients, should not be performed routinely unless concern is triggered in some way about the individual's decision-making ability. On the other hand, previous studies have found that many psychiatric inpatients lack capacity in relation to treatment decisions $[9,10,19]$. Owen et al. found in the same NHS trust as this study that a quarter of all psychiatric inpatients are both informally admitted and lack capacity relating to treatment decisions [20], raising concerns of deprivations of liberty and best interests decisions for such patients. Should all informal inpatients therefore have a routine capacity assessment? The number of assessments would be substantial and already scarce resources would be put under greater pressure if this were implemented. Perhaps, routine capacity assessments should be restricted to informal patients where there may be a deprivation of liberty? However, it has been shown that lawyers and clinicians are unreliable in assessing deprivation of liberty in informal patients without capacity [21,22], in part because the legal concept of deprivation of liberty is unclear, in contrast to mental capacity which is welldefined and reliably assessed in psychiatric inpatients [4]. The guidance suggests all informal patients should have capacity assessed at the start of admission, whether deprivation of liberty is at issue or not, and it seems hard for clinicians to avoid this obligation to ensure the best interests of their inpatients are served. However, this study shows that capacity assessments were documented in only 433 out of 10, 608 informal admissions (4\%), which indicates that mental capacity, let alone deprivation of liberty, is not routinely assessed in practice.

As noted above, one area in which the MHA explicitly requires capacity assessment is in patients who have been receiving medication to treat their mental disorder for three months or more under compulsion. According to section $58 \mathrm{MHA}$, the responsible clinician must assess the presence of an ability to understand the nature, purpose and likely effects of treatment, and if it is absent, or it is present and the patient is refusing, a second opinion doctor is called upon to certify continued treatment without consent (or treatment against a capable refusal). This safeguard was introduced into the 1983 MHA as a safeguard against heavy drug therapy [23]. In this study capacity assessments were recorded in only 507 out of 3740 admissions under section 3 (14\%), and in $23 \%$ (353/1539) of those detained for over 3 months. These finding support concerns raised by the Care Quality Commission that many clinicians are not routinely assessing mental capacity and recording their assessments, and failing their statutory requirement in assessing capacity to consent to ongoing treatment under the MHA [24].

The frequency of incapacity, among those tested for it, was approximately $60 \%$. A higher frequency was seen in patients detained under civil sections of the Mental Health Act (67-84\%), and a lower frequency in informal patients (50\%) consistent with Owen et al's findings [10]. The frequency of incapacity in patients assessed in forensic services was remarkably lower, with only one in five admissions found to lack capacity. In the entire study population, fewer than a quarter of assessments concerned capacity to consent to treatment, but in forensic wards $87 \%$ of assessments were done for this reason. 
This could be linked to better documentation of capacity assessments as part of consent to treatment provisions under Part IV of the MHA to which the majority of forensic inpatients are subjected. It should be noted that only a minority of inpatients in our sample were assessed for capacity and the true prevalence of incapacity cannot be determined from this study.

Incapacity also varied according to diagnosis, with organic and developmental disorders most commonly leading to incapacity. Incapacity was least common in patients with personality disorders, a group in whom assessment of capacity has been found to be complex [25]. Over the study period the proportion of patients found to lack capacity decreased significantly. It is possible that before the implementation of the MCA, capacity was most often assessed only when it was obviously lacking, hence a high proportion of admissions were found to lack capacity. Over the period of the introduction of the MCA there has been a shift in culture, and capacity is being assessed more routinely and this could explain why fewer individuals who are assessed are now found to lack capacity. However, due to the size of the study it was also not possible to look in depth at the reasons why capacity assessments were being done at particular times and on particular patients.

This is the first large scale study looking at the impact of the MCA on clinical practice. One of its main strengths is the size of the sample, which comprises all admissions one of the largest providers of secondary mental healthcare in Europe. The cohort was limited to a single trust in South London, but this is very large trust covers approximately 2\% of the population of England and Wales. We were able to draw on complete electronic clinical records of more than 17,000 inpatients, which provided large amounts of demographic information about each individual, and provided the statistical power to be able to assess the change over time in the assessment of capacity. The disadvantage to using such a large cohort is the lack of qualitative data provided in these results. In order to identify those assessments couched in the appropriate legal terms, capacity assessments were identified only if the term "capacity" was used in the medical records. It is possible that more assessments were carried out but were not documented, or did not use the term "capacity" in the documentation, but due to the large number of patient notes searched in this study it was not feasible to use all potentially relevant search terms, and preliminary searches using the alternative term "competence" yielded no additional documented capacity assessments. It is possible that the use of the term "capacity" also grew during the study period and this could provide further explanation for the rise in documented capacity assessments found in this study.
The assessment of mental capacity can be complex, and the results in this study are limited to what was documented in the electronic notes. The study gives an approximate estimate of when and how capacity was assessed without addressing some of the complexities associated with such assessments. It is likely that capacity assessments are being carried out more frequently and more thoroughly than these results suggest. In a number of cases the records stated the patient lacked capacity without giving reference to an assessment having taken place. The reliance on using the written records makes it difficult to establish whether incapacity was assumed, or whether a full assessment had taken place but was not documented. The electronic records were searched using CRIS, which can access everything in a patient's medical notes other than scanned documents. This means it was not possible to include hand-written forms for documenting capacity assessments, which were available in SLaM during the study period. The study found that such forms were used in only eight cases, but the actual number is likely to be higher.

This study is limited to the assessment of capacity in psychiatric inpatients and it does not look at the impact of the MCA on practice in non-psychiatric settings. While the rates of incapacity are lower in medical than psychiatric patients [8], there are many instances in general medicine and surgery when an individual's capacity to consent to care is impaired. Studies have highlighted a lack of training and knowledge amongst psychiatrists [26] and general hospital doctors [27] with regards to the assessment of mental capacity although it has been shown that psychiatrists fare better than general physicians regarding their knowledge of the MCA [28]. It is likely that incapacity is being overlooked more in nonpsychiatric settings.

The Healthcare Commission (now the CQC) have recommended routine assessment and recording of mental capacity to consent from the start of every inpatient admission [14], but similar to the findings in this study, they have found that this is not happening in practice [24]. This study also shows that clinicians are rarely using, or rarely document using, the MCA criteria for determining capacity. In fewer than $15 \%$ of assessments were the proper criteria for determining mental capacity reported, and in the majority of cases either arbitrary or no criteria were used. While there was a slight improvement in the use of the criteria after the MCA came into force, this suggests there is an ongoing need for training in this area. Studies have shown that capacity can be reliably assessed using a clinical interview and a structured assessment tool [4], while other research has shown that despite the clear guidance in the MCA about how to assess capacity, clinicians have received poor training and lack knowledge of how capacity should be assessed in 
practice $[29,30]$. Along with the limited in-depth training in MCA assessments amongst clinicians, the complexities involved in having an older statute (the MHA), where capacity is not of central significance existing alongside the newer MCA, where it is, may explain this current situation. In addition, the wording of the test for incapacity under section $58 \mathrm{MHA}$ (that the patient is "not capable of understanding the nature, purpose and likely effects of that treatment") differs from the test of capacity set out in the MCA (the patient is unable to understand, retain, or use and weigh information, or cannot communicate their wishes in relation to the decision in question). The existence of two forms of wording for what appears to be the same test is yet another complication that arises from having two statutes.

In this study capacity assessments were predominantly carried out by doctors, and those recorded by approved mental health practitioners mostly concerned the patient's ability to consent to admission as part of a MHA assessment. However, it is important to note that capacity assessments can be carried out by any health or social care practitioner and indeed both the Social Care Institute for Excellence and the British Psychological Society are developing training materials on mental capacity assessment. Prior to the implementation of the MCA it was observed that professionals varied widely in their understanding of capacity legislation and how and when mental capacity should be assessed [31]. More recently a number of small studies have highlighted ongoing deficiencies in clinicians' knowledge and use of the MCA $[28,30]$ despite widely available guidance.

\section{Conclusion}

Over the period of the implementation of the MCA there has been a statistically significant increase in the number of mental capacity assessments being recorded in psychiatric inpatients; however, it is clear that the vast majority of patients are still not routinely assessed for mental capacity. This is a potential source of concern in a population where almost two thirds have previously been found to lack capacity to make decisions relating to their treatment or admission, and suggests that in many cases capacity is not being explicitly clarified. This may result in individuals being given medication, subjected to investigations and procedures, or even deprived of their liberty without clear legal protection, both for the individual and the treating clinicians. In addition, despite the MCA introducing a coherent statutory test for determining incapacity, this is only applied in a minority of cases.

While there appears to be a cultural shift throughout psychiatry and healthcare in general to consider mental capacity as a matter of routine, this apparent necessity to demonstrate capacity in all patients undergoing treatment goes against the first principle of the MCA, that a person must be assumed to have capacity unless it is established that he lacks capacity. This paradox needs to be addressed, and currently there is no clear guidance to assist clinicians in deciding when to document that capacity is present, other than in certain instances where the assessment of capacity is obligatory, such as under section 58 of the MHA. In other circumstances capacity assessments are recommended as routine, such as consent to admission for all informal psychiatric inpatients (although it should be noted that capacity and best interests assessments are obligatory when deprivation of liberty is in question). We recommend that further assessment of capacity should be routine if there is a major change in treatment plan or if significant intervention is recommended, and most importantly all assessments should be documented regardless of whether the patient was found to have or lack capacity. This applies not only to psychiatric patients but also to medical patients, and clinicians should be cautious of discriminating against psychiatric patients by presuming incapacity.

There are few interventions in medicine so ethically complex as treating individuals without their consent yet despite increasing access to information and training, clinical practice in assessing capacity remains poor. The MCA Code of Practice provides some guidance, and where there is a reasonable belief that an individual lacks capacity, as is the case for anybody requiring psychiatric inpatient treatment, this should be sufficient to justify carrying out an assessment. But the resource implications of carrying out and documenting detailed assessments of capacity in all psychiatric inpatients in an already understaffed and overstretched health service are not insignificant and need to be considered.

\section{Unanswered questions and future research}

This study raises a number of questions about how, when and why mental capacity assessments are carried out in practice. Are they done due to an increasingly defensive approach to medical practice? Capacity assessments are increasingly becoming part of a routine consent-gaining procedure, especially for complex treatments such as giving ECT, but are they more likely to be done when a patient is refusing rather than accepting the treatment offered to them? This study does not address the motivation behind assessing capacity, and the effects of ethnicity, diagnosis and other clinical factors on whether concerns about incapacity are raised need to be explored further.

\footnotetext{
Abbreviations

CQC: Care Quality Commission; CRIS: Case Register Interactive Search; ECT: Electroconvulsive therapy; MCA: Mental Capacity Act 2005; MHA: Mental Health Act 1983 (amended 2007); SLaM: South London and Maudsley NHS Foundation Trust.
} 


\section{Competing interests}

All authors have completed the Unified Competing Interest form at www.icmje.org/coi_disclosure.pdf (available on request from the corresponding author) and declare: no support from any organisation for the submitted work

\section{Authors' contribution}

PB designed the study and data collection, monitored data collection, carried out statistical analysis, cleaned and analysed the data, and drafted and revised the paper. She is guarantor. AT carried out data collection, cleaned and analysed the data, and drafted and revised the paper. CM collected data and revised the paper. GO and GS revised the draft paper. $\mathrm{MH}$ initiated and designed the study, oversaw its conduct, and revised the draft paper. All authors read and approved the final manuscript.

\section{Acknowledgements}

The authors would like to thank Prof Genevra Richardson for advice on legal issues raisedin the paper. $\mathrm{MH}, \mathrm{PFB}$ and ADT were supported by the NIHR Biomedical Research Centre for Mental Health at the South London and Maudsley NHS Foundation Trust and Institute of Psychiatry, Kings College London. GSO is funded on a Wellcome Trust Clinical Fellowship in Biomedical Ethics. There are no other relationships or activities that could appear to have influenced the submitted work.

\section{Author details}

${ }^{1}$ Department of Psychological Medicine, Institute of Psychiatry, Weston Education Centre, Cutcombe Road, London SE5 9RJ, UK. ${ }^{2}$ Institute of Psychiatry, Box P068De Crespigny Park, London SE5 8AF, United Kingdom. ${ }^{3}$ Kings College London, School of Medicine, London, UK.

Received: 1 February 2013 Accepted: 11 April 2013 Published: 15 April 2013

\section{References}

1. Mental Capacity Act; 2005

2. Emmett C, Poole M, Bond J, Hughes JC: Homeward bound or bound for a home? Assessing the capacity of dementia patients to make decisions about hospital discharge: Comparing practice with legal standards. Int J Law Psychiatry 2013, 36(1):73-82.

3. Suto WMI, Clare ICH, Holland AJ, Watson PC: Capacity to make financial decisions among people with mild intellectual disabilities. J Intellect Disabil Res 2005, 49(3):199-209.

4. Cairns R, Maddock C, Buchanan A, David AS, Hayward P, Richardson G, Szmukler G, Hotopf M: Reliability of mental capacity assessments in psychiatric in-patients. Br J Psychiatry 2005, 187:372-378

5. Owen GS: Mental capacity and psychopathology. Psychiatry 2009, 8(12):476-477

6. Ranjith G, Hotopf M, Ranjith G, Hotopf M: 'Refusing treatment-please see': an analysis of capacity assessments carried out by a liaison psychiatry service. J R Soc Med 2004, 97(10):480-482.

7. Umapathy C, Ramchandani D, Lamdan RM, Kishel LA, Schindler BA: Competency evaluations on the consultation-liaison service. Some overt and covert aspects. Psychosomatics 1999, 40(1):28-33.

8. Raymont V, Bingley W, Buchanan A, David AS, Hayward P, Wessely S, Hotopf M: Prevalence of mental incapacity in medical inpatients and associated risk factors: Cross-sectional study. Lancet 2004, 364(9443):1421-1427.

9. Cairns R, Maddock C, Buchanan A, David AS, Hayward P, Richardson G, Szmukler G, Hotopf M: Prevalence and predictors of mental incapacity in psychiatric in-patients. Br J Psychiatry 2005, 187(4):379-385.

10. Owen GS, Richardson G, David AS, Szmukler G, Hayward P, Hotopf M, Owen GS, Richardson G, David AS, Szmukler G, et al: Mental capacity to make decisions on treatment in people admitted to psychiatric hospitals: cross sectional study. BMJ 2008, 337:a448.

11. Department of Health Mental Health Act 1983: Code of Practice. London: The Stationary Office; 2008

12. Care Quality Commission: Monitoring the Mental Health Act in 2010/11: The Care Quality Commission's annual report on the exercise of its functions in keeping under review the operation of the Mental Health Act 1983; 2011.

13. Mental Health Act; 1983.

14. Healthcare Commission: The Pathway to Recovery - A review of NHS acute inpatient mental health services; 2008.
15. Care Quality Commission: 13th biennial report of Mental Health Act Commission; 2009.

16. Chang CK, Hayes RD, Broadbent M, Fernandes AC, Lee W, Hotopf M, Stewart R: All-cause mortality among people with serious mental illness (SMI), substance use disorders, and depressive disorders in southeast London: A cohort study. BMC Psychiatry 2010, 10:77. doi:10.1186/1471244X-10-77.

17. Stewart R, Soremekun M, Perera G, Broadbent M, Callard F, Denis M, Hotopf M, Thornicroft G, Lovestone S: The South London and Maudsley NHS Foundation Trust Biomedical Research Centre (SLAM BRC) case register: development and descriptive data. BMC Psychiatry 2009, 9:51.

18. Tulloch AD, Fearon P, David AS: Residential mobility among patients admitted to acute psychiatric wards. Health Place 2011, 17(4):859-866

19. Okai D, Owen G, McGuire H, Singh S, Churchill R, Hotopf M: Mental capacity in psychiatric patients: Systematic review. Br J Psychiatry 2007 191(2):291-297.

20. Owen GS, Szmukler G, Richardson G, David AS, Hayward P, Rucker J, Harding D, Hotopf M: Mental capacity and psychiatric in-patients: Implications for the new mental health law in England and Wales. Br J Psychiatry 2009, 195(3):257-263.

21. Cairns R, Brown P, Grant-Peterkin H, Owen GS, Richardson G, Szmukler G, Hotopf M: Mired in confusion: making sense of the Deprivation of Liberty Safeguards. Med Sci Law 2011, 51(4):228-236.

22. Cairns R, Brown P, Grant-Peterkin H, Khondoker MR, Owen GS, Richardson G, Szmukler G, Hotopf M: Judgements about deprivation of liberty made by various professionals: comparison study. Psychiatrist 2011, 35(9):344-349.

23. Fennell P: Treatment Without Consent: law, psychiatry and the treatment of mentally disordered people since 1845. London: Routledge; 1996.

24. Care Quality Commission: Monitoring the use of the Mental Health Act in 2009/10. In The Care Quality Commission's first report on the exercise of its functions in keeping under review the operation of the Mental Health Act 1983; 2010.

25. Szmukler G: "Personality disorder" and capacity to make treatment decisions. J Med Ethics 2009, 35(10):647-650

26. Hill SA, Mather Gl, James AJ, Hill SA, Mather GI, James AJB: Assessing decision-making capacity: a survey of psychiatrists' knowledge. Med SCi Law 2006, 46(1):66-68.

27. Richards F, Dale J: The Mental Health Act 1983 and incapacity: What general hospital doctors know. Psychiatr Bull 2009, 33(5):176-178.

28. Schofield C: Mental Capacity Act 2005-what do doctors know? Med Sci Law 2008, 48(2):113-116.

29. Shah A, Banner N, Heginbotham C, Fulford B, Shah A, Banner N, Heginbotham C, Fulford B: A pilot study of the early implementation of the Mental Capacity Act 2005 in England and Wales: the experience of consultants in old age psychiatry. Med Sci Law 2010, 50(3):131-135.

30. Willner P, Jenkins R, Rees P, Griffiths VJ, John E: Knowledge of mental capacity issues in community teams for adults with learning disabilities. J Appl Res Intellect Disabil 2011, 24(2):159-171.

31. Myron RGS, Swift P, Williamson T: Whose decision? Preparation for and Implementation of the Mental Capacity Act in Statutory and Non-statutory Services in England and Wales. London: Mental Health Foundation; 2008.

doi:10.1186/1471-244X-13-115

Cite this article as: Brown et al:: Assessments of mental capacity in psychiatric inpatients: a retrospective cohort study. BMC Psychiatry 2013 13:115 